\title{
Zum Anliegen der RECHTSWISSENSCHAFT
}

\author{
Johannes Rux*
}

\section{A. Die Rechtswissenschaft befindet sich in der Krise.}

Die juristische Ausbildung wird nach wie vor vom Idealtypus des universell einsetzbaren Volljuristen geprägt, der mit Hilfe der juristischen Auslegungsmethoden auf jede Rechtsfrage eine Antwort finden kann. Tatsächlich wird dieses Ideal - wenn überhaupt - nur noch von denjenigen Anwälten gelebt, die es sich nicht leisten können oder wollen, Mandate abzulehnen und daher zumindest im Grundsatz in allen Rechtsgebieten gleichermaßen zuhause sind. Auch diese Feld-Wald-und-Wiesen-Anwälte stoßen bei komplexeren Sachverhalten jedoch schnell an ihre Grenzen und sind daher gezwungen, ihre Mandanten an Spezialisten zu verweisen. Selbst in Justiz und Verwaltung sind wahre Volljuristen eher die Ausnahme als die Regel. Zwar versuchen viele Dienstherren, der Tendenz zur Spezialisierung dadurch entgegen zu wirken, dass teilweise auch die fachliche Mobilität zur Voraussetzung für den Aufstieg in Beförderungsämter gemacht wird. In der Regel bedeutet dies jedoch nur, dass Bewerber schon einmal das Dezernat gewechselt haben müssen. Denn schließlich ist auch hier eine gewisse Spezialisierung notwendige Voraussetzung für die Bearbeitung komplexer Rechtsfragen.

In der Tat hat die zunehmende Komplexität der Lebensverhältnisse zu einer Ausdifferenzierung der Rechtsordnung geführt, die sich ab einem bestimmten Niveau nur noch von Spezialisten beherrschen lässt. Neben das klassische staatliche Recht, dessen Komplexität trotz aller Bemühungen um Deregulierung und Entbürokratisierung stetig zunimmt, sind überstaatliche Rechtsnormen und teilweise auch die Regelwerke privater Organisationen getreten, die für die Betroffenen zumindest faktisch ebenso verbindlich sind wie Parlamentsgesetze.

Die Notwendigkeit zur Spezialisierung spiegelt sich selbstverständlich auch in der Rechtswissenschaft als akademischer Disziplin. Zwar ist die Unterscheidung zwischen den großen Gebieten des Öffentlichen, Straf- und Zivilrechts keineswegs neu. In den letzten Jahren oder eigentlich schon Jahrzehnten scheinen sich die Grenzen zwischen diesen Rechtsgebieten jedoch immer weiter verfestigt zu haben und selbst innerhalb der großen Rechtsgebiete droht allmählich der Zusammenhang verloren zu gehen. Selbst in den Grundlagenfächern bleibt der wissenschaftliche Diskurs nur allzu oft rechtsgebietsbezogen.

Spezialisierung setzt den Zugriff auf diejenigen Informationen voraus, die für das eigene Sujet von Bedeutung sind. Da die Menge dieser Informationen ständig wächst hat die Spezialisierung auch zu einer Ausdifferenzierung der Informationsmedien ge-

* PD Dr. Johannes Rux lehrt Öffentliches Recht an der Eberhard-Karls-Universität Tübingen und ist Schriftleiter der RECHTSWISSENSCHAFT. 
führt. Umgekehrt befördert die geradezu epidemische Verbreitung juristischer Fachzeitschriften die Tendenz zur Spezialisierung, da selbst der neugierigste Leser nur eine bestimmte Zahl von Fachzeitschriften zur Kenntnis nehmen kann. Wenn es nun aber selbst hochmotivierten Hochschullehrern nicht mehr möglich ist, den Überblick über die wichtigsten Entwicklungen innerhalb der anderen Rechtsgebiete zu behalten, stellt dies nicht nur das Ideal des Volljuristen und damit das Leitmotiv der rechtswissenschaftlichen Lehre in Frage. Vielmehr droht auch eine Fragmentierung der Forschung: Während auf der einen Seite völlig zu Recht Interdisziplinarität und die enge Kooperation mit Nachbardisziplinen gefordert und gepflegt wird, droht auf der anderen Seite gleichzeitig der Zusammenhalt zwischen den Teildisziplinen der Jurisprudenz verloren zu gehen. Auf lange Sicht scheint es dann aber nicht ausgeschlossen, dass die Rechtswissenschaft ihre Eigenständigkeit verlieren und zur Hilfswissenschaft anderer Disziplinen verkümmern könnte - und zwar völlig unabhängig davon, dass das Recht als Erkenntnisgegenstand nichts von seiner Faszination verloren hat.

So unwahrscheinlich eine solche Entwicklung aus heutiger Perspektive erscheinen mag, ist sie doch nicht völlig ausgeschlossen. Dies gilt umso mehr als die wichtigsten Garanten für die Eigenständigkeit der Rechtswissenschaft als akademischer Disziplin immer lauter in Frage gestellt werden: Nämlich das juristische Staatsexamen als notwendige Voraussetzung für die Ausübung qualifizierter juristischer Berufe und das darin begründete faktische Ausbildungsmonopol der juristischen Fakultäten. Tatsächlich dürfte genau hier ein wichtiges wenn nicht gar das zentrale Motiv für den beharrlichen Widerstand gegen die Umstellung der juristischen Ausbildung auf Bachelor- und Masterstudiengänge zu suchen sein.

\section{B. Die RECHTSWISSENSCHAFT führt aus der Krise}

Natürlich ist die Lage doch noch nicht ganz so ernst: Viele Hochschullehrer suchen den Kontakt zu Kollegen aus den anderen Gebieten - und erleben dabei immer wieder, dass sich teilweise durchaus unerwartet Berührungspunkte ergeben. Offensichtlich ist dies bei der Beschäftigung mit den Grundlagen des Rechts und bei Querschnittsthemen wie dem Einfluss des Völker- und Europarechts auf die nationale Rechtsordnung. Aber auch in vielen anderen Bereichen werden bei näherer Betrachtung Beziehungen deutlich, die auch für die eigene wissenschaftliche Arbeit nutzbar gemacht werden können.

Mit der neuen Zeitschrift RECHTSWISSENSCHAFT wollen wir ein Gegengewicht zur zunehmenden Spezialisierung schaffen und die Zusammenhänge zwischen den rechtswissenschaftlichen Teildisziplinen aufzeigen. Dabei soll es nicht allein den Lesern überlassen bleiben, Bezüge herzustellen. Vielmehr sollen diese Bezüge in jedem einzelnen Beitrag deutlich gemacht werden. Die neue Zeitschrift will auf diese Weise eine Klammer bilden, die den rechtswissenschaftlichen Diskurs in den einzelnen Dis- 
ziplinen zusammenführt. Die Beiträge stellen auf der einen Seite den Stand der Forschung auf höchstem Niveau dar und geben damit auf der anderen Seite den Lesern unverzichtbare Anregungen für ihre eigene Tätigkeit in Forschung und Lehre.

Die RECHTSWISSENSCHAFT richtet sich in erster Linie an Hochschullehrer und den wissenschaftlichen Nachwuchs, aber auch an andere Personen, die sich in Forschungsinstituten, Ministerien, Gerichten, Verbänden, Unternehmen oder Anwaltskanzleien auf höchstem wissenschaftlichem Niveau mit rechtswissenschaftlichen Themen auseinandersetzen.

\section{Die Umsetzung}

Das soeben formulierte hochgesteckte Ziel hat uns dazu bewogen, die traditionellen Formate juristischer Fachzeitschriften ebenso grundsätzlich in Frage zu stellen, wie die herkömmlichen Instrumente der Qualitätskontrolle.

\section{Die Formate}

Der fachgebietsübergreifende Anspruch der RECHTSWISSENSCHAFT und ihre Ausrichtung auf Forschung und Lehre führen dazu, dass die klassischen Formate juristischer Beiträge etwas modifiziert werden müssen.

\section{Abhandlungen}

Da sich die RECHTSWISSENSCHAFT nicht nur an Spezialisten für ein bestimmtes Rechtsgebiet richtet, kann nicht ohne Weiteres vorausgesetzt werden, dass alle Leser den Stand der Forschung überblicken. Daher werden größere Abhandlungen gegebenenfalls mit einer Einleitung versehen, in der das Problem und der Zusammenhang innerhalb der engeren Fachdiskussion auf eine - für Juristen - allgemeinverständliche Weise dargestellt werden. Diese Einleitung ist keine ausführliche Darstellung des Forschungsstandes und kann daher weitgehend ohne legitimatorische Fußnoten auskommen.

Die ersten beiden großen Abhandlungen in Heft 1 der RECHTSWISSENSCHAFT können auf eine solche Einführung verzichten, da sie sich mit Grundsatzfragen befassen, die jedem Rechtswissenschaftler zumindest ansatzweise vertraut sind. Horst Dreier stellt in seinem einleitenden Beitrag die These auf, dass der freiheitliche Verfassungsstaat als riskante Ordnung auf alle Fundamentierungen verzichten kann und muss, die jenseits des Willens zur Selbstbestimmung liegen. Nach seiner Ansicht haben Grundpflichten in der Kombination aus politischer und personaler Autonomie keinen Platz. Den Kitt, den der freiheitliche Verfassungsstaat brauche, liefern nicht Religion oder Verfassungspatriotismus, sondern der Dissens. Gerade deshalb benötige der freiheitliche Verfassungsstaat ein Minimum an sozialer Homogenität in Form einer gemeinsamen Sprache - und Toleranz als Bürgertugend, die jedem an- 
deren den Genuss seiner gleichen Freiheit lässt und die hieraus erwachsenden $\mathrm{Zu}$ mutungen erträgt.

Thomas Weigend beschäftigt sich mit den strafrechtstheoretischen Implikationen der Debatte über die Stellung des Opfers im Strafrecht und Strafverfahrensrecht. Aus dem Allgemeinen Persönlichkeitsrecht leitet er einen differenzierten Anspruch des Opfers auf Durchführung eines Strafverfahrens bzw. auf Bestrafung „seines“ Täters ab. Diese verfassungsrechtliche Begründung wirkt sich auf das Strafverfahrensrecht aus und wirft die Frage auf, ob und in wie weit die Bestimmungen über die Nebenklage den Ansprüchen des Opfers gerecht werden.

Der Beitrag von Helge Dedek über „Die Schönheit der Vernunft“ beschäftigt sich nur auf den ersten Blick mit einer Spezialmaterie der Rechtsgeschichte. Wie Dedek in seiner Einleitung deutlich macht, kann die Auseinandersetzung mit der scheinbaren Irrationalität der mittelalterlichen Rechtswissenschaft unsere Augen dafür öffnen, dass auch der modernen Rechtswissenschaft irrationale Elemente inne wohnen. Diese Erkenntnis erleichtert uns aber nicht nur den Blick zurück in die Rechtsgeschichte sondern auch die Rechtsvergleichung, bei der es eben nicht nur auf den jeweiligen Rechtstext ankommt sondern auch auf den kulturell geprägten Habitus der Rechtsanwender.

\section{Rezensionen und Tagungsberichte}

Im Rezensionsteil geht es nicht nur darum, den Inhalt herausragender Veröffentlichungen darzustellen. Vielmehr soll das Thema aufgegriffen und weiter gesponnen werden, um den wissenschaftlichen Diskurs auf diese Weise zu befördern. In Heft 1 der Rechtswissenschaft stellt Michael Sachs die Frage, wie die Habilitation der österreichischen Kollegin Magdalena Pöschl über die Gleichheit vor dem Gesetz auch die entsprechende Diskussion in Deutschland beeinflussen kann. Christian Jäger setzt sich auf Grundlage der Habilitationsschrift von Armin Engländer über Grund und Grenzen der Nothilfe auseinander - einer Thematik, die Übrigens auch für das Öffentliche Recht eine große Rolle spielt.

In eine ganz ähnliche Richtung wie die Rezensionsaufsätze zielen die Tagungsberichte, bei denen es sich tatsächlich um eine Art Ko-Referat handelt: Auch hier geht es darum, die durch die Referate auf einer Tagung angestoßene Diskussion aufzunehmen und fortzuführen - und dabei nach Möglichkeit zugleich Bezüge zu anderen Rechtsgebieten herzustellen oder allgemeine Fragen aufzuwerfen. In Heft 1 tut dies Werner Heun am Beispiel der Grazer Staatsrechtslehrertagung im Oktober 2009.

\section{Die Rahmenbedingungen von Forschung und Lehre}

Eine Zeitschrift, die sich in erster Linie an Hochschullehrer und den wissenschaftlichen Nachwuchs richtet, muss selbstverständlich auch die Rahmenbedingungen von Forschung und Lehre im Auge behalten. Hier geht es nicht nur um die Darstellung 
neuerer Entwicklungen sondern vielmehr um die kritische Reflexion und darum, Perspektiven für die Rechtswissenschaft zu eröffnen. Eine große Rolle spielt dabei unter anderem die Diskussion über die Umsetzung der Bologna-Empfehlungen in der juristischen Ausbildung, der Thomas Pfeiffer in Heft 1 seine Aufmerksamkeit widmet.

\section{Die Qualitätskontrolle}

Um die Qualität der Beiträge zu gewährleisten, haben wir uns gleich doppelt abgesichert: Auf der einen Seite wacht ein Kreis aus 15 Herausgeberinnen und Herausgebern über die Qualität der Texte. Auf der anderen Seite übernimmt die RECHTSWISSENSCHAFT internationale Standards für wissenschaftliche Zeitschriften und sieht eine echte Peer Review mit anonymer Begutachtung durch Fachkollegen vor.

\section{Die Herausgeber}

Wir freuen uns, dass es uns gelungen ist, 15 herausragende Kolleginnen und Kollegen, die mit ihren jeweiligen Spezialgebieten die Rechtswissenschaft in ihrer ganzen Breite spiegeln, für den Herausgeberkreis der RECHTSWISSENSCHAFT zu gewinnen. Bei einer „normalen“ Fachzeitschrift sollte man davon ausgehen, dass die Namen der Herausgeberinnen und Herausgeber für sich stehen. Aufgrund der oben beschriebenen Ausdifferenzierung der Rechtswissenschaft kann man heute jedoch nicht einmal bei Hochschullehrern davon ausgehen, dass jeder einen Überblick über die Nachbardisziplinen hat. Aus diesem Grund sollen die Herausgeber der RECHTSWISSENSCHAFT hier zumindest mit ihren wichtigsten Funktionen vorgestellt werden. Zu dem Kreis gehören:

- Prof. Dr. Barbara Dauner-Lieb, Inhaberin des Lehrstuhls für Bürgerliches Recht, Handels- und Gesellschaftsrecht, Arbeitsrecht und Europäische Privatrechtsentwicklung, Direktorin des Instituts für Arbeits- und Wirtschaftsrecht und des Instituts für Gesellschaftsrecht der Universität zu Köln, Richterin am Verfassungsgerichtshof für das Land Nordrhein-Westfalen, Mitglied des DFG-Fachkollegiums Rechtswissenschaft

- Prof. Dr. Hans Christoph Grigoleit, Inhaber des Lehrstuhls für Bürgerliches Recht, Handels- und Gesellschaftsrecht, Europäisches Privatrecht, Universität Regensburg

- Prof. Dr. Thomas Gutmann, Inhaber des Lehrstuhls für Bürgerliches Recht, Rechtsphilosophie und Medizinrecht, Westfälische Wilhelms-Universität Münster

- Prof. Dr. Hans-Peter Haferkamp, Inhaber des Lehrstuhls für Bürgerliches Recht, Neuere Privatrechtsgeschichte und Deutsche Rechtsgeschichte, Direktor des Instituts für Neuere Privatrechtsgeschichte, Deutsche und Rheinische Rechtsgeschichte der Universität zu Köln 
- Prof. Dr. Dr. h.c. Werner Heun, Leiter des Instituts für Allgemeine Staatslehre und Politische Wissenschaften der Georg-August-Universität Göttingen

- Prof. Dr. Tatjana Hörnle, Inhaberin des Lehrstuhls für Strafrecht, Strafprozessrecht, Rechtsphilosophie und Rechtsvergleichung der Humboldt-Universität zu Berlin, Mitglied des DFG-Fachkollegiums Rechtswissenschaft

- Prof. Dr. Abbo Junker, Inhaber des Lehrstuhls für (Internationales) Arbeitsrecht, Arbeitsrechtsvergleichung und Bürgerliches Recht, Direktor der Abteilung II Europäisches und Internationales Arbeitsrecht sowie Systemvergleich des Zentrums für Arbeitsbeziehungen und Arbeitsrecht der Ludwig-Maximilians-Universität München

- Prof. Dr. Hans-Jürgen Kerner, Inhaber des Lehrstuhls für Kriminologie, Jugendstrafrecht, Strafvollzug und Strafprozessrecht, Direktor des Instituts für Kriminologie der Eberhard-Karls-Universität Tübingen, Mitglied des DFG-Fachkollegiums Rechtswissenschaft

- Prof. Dr. Dr. h.c. Urs Kindhäuser, Inhaber des Lehrstuhls für Strafrecht und Strafprozessrecht, Geschäftsführender Direktor des Instituts für Strafrecht der Universität Bonn

- Prof. Dr. Anne Peters LL.M., Inhaberin des Lehrstuhls für Völker- und Staatsrecht, Universität Basel

- Prof. Dr. Thomas Pfeiffer, Inhaber des Lehrstuhls für Bürgerliches Recht, Internationales Privatrecht, Rechtsvergleichung, Internationales Verfahrensrecht, Direktor des Instituts für ausländisches und internationales Privat- und Wirtschaftsrecht der Ruprecht-Karls-Universität Heidelberg

- Prof. Dr. Helmut Satzger, Inhaber des Lehrstuhls für Deutsches, Europäisches und Internationales Strafrecht und Strafprozessrecht sowie Wirtschaftsstrafrecht, Ludwig-Maximilians-Universität München

- Prof. Dr. Helmuth Schulze-Fielitz, Lehrstuhl für Öffentliches Recht, Umweltrecht und Verwaltungswissenschaften, Julius-Maximilians-Universität Würzburg und bis vor kurzem Vorsitzender der Vereinigung der Deutschen Staatsrechtslehrer

- Prof. Dr. Ewald Wiederin, Inhaber des Lehrstuhls für Staats- und Verwaltungsrecht, Universität Wien

- Prof. Dr. Joachim Wieland, Inhaber des Lehrstuhls für Öffentliches Recht, Finanzund Steuerrecht, Deutsche Hochschule für Verwaltungswissenschaften Speyer, Mitglied des Verfassungsgerichtshofs für das Land Nordrhein-Westfalen

\section{Das Peer-Review-Verfahren}

Die RECHTSWISSENSCHAFT ist eine Peer-Review-Zeitschrift. Wir wollen damit zum einen ein zusätzliches Instrument zur Qualitätssicherung einsetzen, das - wie schon bei den Vorbereitungen für das Heft 1 deutlich wurde - zugleich wertvolle Anregungen für die Autoren liefern kann. Zum anderen tragen wir damit interna- 
tionalen Standards Rechnung, da sich das in den deutschsprachigen Ländern übliche System der Qualitätskontrolle durch die Herausgeber trotz seiner unbestreitbaren Qualitäten international nicht durchgesetzt hat. Wer mit Fachkollegen aus dem Ausland zu tun hat, wird daher immer wieder mit der Frage konfrontiert, in welchen Peer-Review-Zeitschriften man denn schon veröffentlicht habe - wobei nicht selten fast im selben Atemzug die Schwerfälligkeit des Peer-Review-Verfahrens moniert wird. Wir haben versucht, beide Modelle der Qualitätskontrolle sinnvoll miteinander zu kombinieren. Das Verfahren läuft folgendermaßen ab:

Beiträge für Abhandlungen werden von den Autoren oder auf Anfrage der Herausgeber eingereicht. Sie werden zunächst an die jeweiligen Fachkollegen innerhalb des Herausgebergremiums weiter geleitet. Diese haben das Recht, einen Beitrag a limine abzuweisen, wenn dieser den Qualitätsanforderungen oder dem Konzept der Zeitschrift nicht entspricht. Andernfalls bestimmen sie einen Berichterstatter, der den Text an die Schriftleitung zurückgibt.

Von der Schriftleitung werden die Texte anonymisiert und an zwei Gutachter übergeben. Über die Auswahl der Gutachter entscheidet die Schriftleitung nach Absprache mit dem Berichterstatter und ggf. weiteren Herausgebern. Den Gutachtern wird ein Fragebogen mitgegeben, auf dem sie Stellung zu dem Beitrag nehmen können.

Die Gutachten werden an alle Herausgeber geleitet, die auf dieser Grundlage entscheiden, ob

- der Beitrag unverändert erscheint,

- der Autor vor der Veröffentlichung um Änderungen oder Ergänzungen gebeten werden soll,

- der Beitrag endgültig abgelehnt wird.

Haben die Gutachter einheitlich für die Annahme oder Ablehnung eines Beitrags votiert, gilt das Schweigen der Herausgeber als Zustimmung zu diesem Votum. In allen anderen Fällen bedarf es der ausdrücklichen Zustimmung bzw. Ablehnung durch mindestens die Hälfte der Herausgeber. Für die Entscheidung über die Annahme bzw. Ablehnung eines Beitrags und für Stellungnahmen zu den Gutachten gilt grundsätzlich eine Frist von vier Wochen.

Wurde ein Beitrag abgelehnt, erhält der Verfasser den Text mit einem durch die Schriftleitung bearbeiteten und anonymisierten Auszug aus den Gutachten zurück. Dasselbe gilt dann, wenn die Gutachter oder die Herausgeber Änderungen vorgeschlagen haben. In diesem Fall wird dem Autor eine angemessene Frist für die Überarbeitung gesetzt. Reicht er den geänderten Beitrag fristgerecht wieder ein, entscheiden die Herausgeber im Umlaufverfahren über die Annahme des Manuskriptes.

In Heft 4 jedes Jahrgangs werden die Gutachter des vergangenen Jahrs namentlich genannt. 


\section{Schluss}

Natürlich freuen wir uns über Kritik und Anregungen und hoffen, dass wir Sie mit diesem Heft auf die nächsten Ausgaben neugierig machen. Mit der Veröffentlichung des ersten Heftes fängt für uns die eigentliche Arbeit erst an: Denn nun geht es darum, die nächsten Ausgaben zu füllen. Wir sind überzeugt, dass es genügend Kolleginnen und Kollegen gibt, die nicht nur am Blick über den Tellerrand interessiert sind sondern auch in ihrer eigenen Arbeit Bezüge zwischen den Teildisziplinen der Rechtswissenschaft herstellen und die neue Zeitschrift RECHTSWISSENSCHAFT als Forum nutzen werden. Beiträge für die RECHTSWISSENSCHAFT können jederzeit bei der Schriftleitung eingereicht werden. Die Kontaktdaten finden Sie unten auf Seite 2 des Heftes. Hinweise für die Manuskriptgestaltung sind auf der Internet-Seite www.rechtswissenschaft.nomos.de abrufbar. 